\title{
A GALERKIN METHOD FOR CALCULATION OF LAMINAR THERMAL BOUNDARY LAYERS
}

\author{
W. P. Graebel \\ Department of Mechanical Engineering and Applied Mechanics, \\ The University of Michigan, Ann Arbor, MI 48109, U.S.A. \\ and \\ C. C. Hsu \\ Department of Engineering Sciences, The University of Florida, Gainesville, FL 32611, U.S.A.
}

(Received 26 March 1980 and in revised form 23 September 1980)

\section{NOMENCLATURE}

$C_{P}, \quad$ specific heat at constant pressure

$g$, gravitational acceleration;

$k$, thermal diffusivity;

$L_{k}^{2 / 3}$, generalized Laguerre polynomial;

$\mathrm{Pr}, \quad$ Prandtl number;

$q$, dimensionless heat flux parameter;

Re, Reynolds number;

$T_{1}, \quad$ dimensional temperature;

$T_{R}$, reference temperature;

$T_{w}, \quad$ wall temperature;

$T_{\infty}, \quad$ temperature outside of boundary layer;

$u_{1}, v_{1}$, dimensional $x_{1}$ and $y_{1}$ velocity components;

$U_{1}$, dimensional $x_{1}$ velocity component outside of boundary layer;

$U$, dimensionless $x$ velocity component outside of boundary layer;

$w, \quad$ square of dimensionless $x$ velocity component;

$x_{1}, y_{1}$, tangential and normal dimensional coordinates;

$x$ transformed dimensional length.

Greek symbols

$\alpha_{1}$, angle between the direction of gravity and the local tangent to the boundary;

$\beta_{1}$, thermal expansivity;

$\rho$, mass density;

$\tau, \quad$ dimensionless wall shear;

$\tau_{w}, \quad$ dimensional wall shear;

$\mu$, viscosity;

$\phi$, dimensionless temperature parameter :

$\Phi$, dimensionless temperature parameter outside of boundary layer.

TWo ESSENTIAL classes of methods have evolved for the solution of boundary layer flows: discretization methods, and weighted residual methods. In the class of weighted residual methods, with which the work in this study is concerned, a solution is sought using a family of basis functions to generate a truncated series. The selection of this set of basis functions is crucial in terms of both accuracy and rate of convergence of the truncated series. A formulation which anticipates this problem and minimizes it may be expected to yield a significant improvement in the effectiveness of the algorithm. Such a consideration was the background for the work of Hsu [1] who presented as a basis a complete set of orthogonal eigenfunctions which are particularly pertinent to the governing boundary-layer equations for a class of steady two-dimensional incompressible laminar flow. The basis functions have closed form solution, and can be predetermined and used in a
Galerkin method for all problems governed by the system of equations treated. This is advantageous, for although complete sets of orthogonal eigenfunctions have been previously employed [2-4], the sets used were particular to the perturbations about a given Falkner-Skan solution and were to be determined numerically in each case. By further ensuring the satisfaction of certain compatability conditions Hsu demonstrated that the formulation in terms of the set of basis function can efficiently reproduce accurate results over large regions of the boundary layer for two standard flow problems. The purpose of this study is to examine whether or not the method and its formulations may be adapted with similar advantage to deal with flows involving the additional complications of heat transfer, in forced and free convection.

For a steady, two-dimensional, incompressible, laminar flow past a submerged body, the governing boundary-layer equations in dimensional form are

$$
\begin{aligned}
& \frac{\partial u_{1}}{\partial x_{1}}+\frac{\partial v_{1}}{\partial y_{1}}=0 \\
& \rho\left(u_{1} \frac{\partial u_{1}}{\partial x_{1}}+v_{1} \frac{\partial u_{1}}{\partial y_{1}}\right) \\
& =\rho U_{1} \frac{\mathrm{d} U_{1}}{\mathrm{~d} x_{1}}+\mu \frac{\partial u_{1}^{2}}{\partial y_{1}^{2}}+\rho g \beta_{1}\left(T_{1}-T_{R}\right) \cos \alpha_{1}, \\
& \rho C_{p}\left(u_{1} \frac{\partial T_{1}}{\partial x_{1}}+v_{1} \frac{\partial T_{1}}{\partial y_{1}}\right) \\
& =k \frac{\partial^{2} T}{\partial y_{1}^{2}}+\mu\left(\frac{\partial u_{1}}{\partial y_{1}}\right)^{2}+\rho C_{p} u_{1} \frac{\mathrm{d} T_{\infty}}{\mathrm{d} x_{1}} .
\end{aligned}
$$

The boundary conditions are

$$
\begin{aligned}
& u_{1}\left(x_{1}, 0\right)=0, \quad v_{1}\left(x_{1}, 0\right)=0, \quad T_{1}\left(x_{1}, 0\right)=T_{w}\left(x_{1}\right), \\
& u_{1}\left(x_{1}, \infty\right)=U_{1}\left(x_{1}\right), \quad T_{1}\left(x_{1}, \infty\right)=T_{\infty}\left(x_{1}\right) .
\end{aligned}
$$

The number of dependent parameters can be reduced by a transformation of von Mises type, and the rate of variation of the boundary-layerathickness can be minimized by a Falkner-Skan transformation, according to

$$
\begin{aligned}
& x=\int_{0}^{x_{1}} U_{1}(s) \mathrm{d} s / L U_{\infty}, \quad \eta=x^{-1 / 4}\left[\int u_{1}\left(x_{1}, s\right) \mathrm{d} s / U_{1}\right]^{1 / 2} \\
& w=\left(u_{1} / U_{1}\right)^{2}, \quad v=y_{1} u_{1} \frac{\mathrm{d} \ln U_{1}}{\mathrm{~d} x} /\left(v L U_{\infty}\right)^{1 / 2}+v_{1}\left(L U_{\infty} / v\right)^{1 / 2} / U_{1}, \\
& U=U_{1} / U_{\infty}, \quad \phi=\left(\left(T_{1}-T_{w}\right) /\left(T_{\infty}-T_{w}\right)\right)^{2}
\end{aligned}
$$

$$
\Phi=\left(T_{\infty}-T_{w}\right) / T_{R} \text {. }
$$


In terms of these variables the equations become

$$
\begin{aligned}
& x \frac{\partial w}{\partial x} \\
& =\frac{w^{1 / 2}}{4 \eta^{2}}\left(\frac{\partial^{2} w}{\partial \eta^{2}}-\frac{1 \partial w}{\eta \partial \eta}\right)+\frac{\eta \partial w}{4 \partial \eta}+\beta(1-w)+A\left(\phi^{12}-1\right),(6) \\
& x \frac{\partial \phi}{\partial x}=\frac{w^{12}}{4 P r \eta^{2}}\left[\frac{\partial^{2} \phi}{\partial \eta^{2}}-\frac{1 \partial \phi}{\eta \partial \eta}+\frac{1 \partial \ln (w / \phi)}{2} \frac{\partial \phi}{\partial \eta}\right] \\
& +\frac{\eta \partial \phi}{4 \hat{\partial \eta \eta}}+B\left(\phi^{12}-\phi\right)+C(\phi / w)^{1 / 2}(\partial w / \eta \hat{\partial} \eta)^{2}
\end{aligned}
$$

$w(x, 0)=0, \quad \phi(x, 0)=0, \quad w(x, x)=1, \quad \phi(x, x)=1,(8)$ where the following notation has been introduced:

$$
\beta(x)=2 x \mathrm{~d} \ln U / \mathrm{d} x, \quad A(x)=2 x \Phi g \beta_{1} L T_{R} \cos \alpha_{1} / U^{3} U_{x}^{2} .
$$

$$
B(x)=2 x \mathrm{~d} \ln \Phi / \mathrm{d} x, \quad C(x)=U^{2} U_{x}^{2} / 8 \Phi C_{p} T_{R}
$$

The case of free convection, where $U_{1}$ is identically zero, can be treated in a similar fashion by making the transformations

$$
x=\int_{0}^{x_{1}} f(s) \mathrm{d} s / L, \quad w=\left(u_{1} L / v f\right)^{2} .
$$

The result will be equations (6) and (7) with only the definitions of the parameters $\beta, A$ and $C$ altered. Now

$\beta=0, \quad A=2 x \Phi g \beta_{1} L^{3} T_{R} \cos \alpha_{1} / f^{3} v^{2}$

$$
C-v^{2} f^{2} / 8 C_{p} L^{2} T_{R} \Phi \text {. }
$$

The dimensionless function $f$ can be defined in a manner convenient to the problem at hand. An obvious choice would be to define it so that $A$ is unity. For free convection, boundary condition (8) would be replaced by one where $w$ vanishes as $\eta$ approaches infinity.

The Galerkin technique proposed by Hsu uses an orthogonal set of representing functions which by themselves satisfy the dominant terms in the equations. In this spirit, the equations are first brought into a form which emphasizes the dominant terms by a further change of independent variable replacing $\eta$ by $\check{\zeta}$, where

$$
\zeta=\left[\int_{0}^{\pi} s w_{0}^{-1 / 4}(s) \mathrm{d} s\right]^{2}
$$

in which $w_{0}$ is the Falkner-Skan solution, that is, the solution of equation (6) with only $\eta$ dependency. Under this transformation, equations (6) and (7) become

$$
\begin{aligned}
x \frac{\partial w}{\partial x}= & \zeta \frac{\partial^{2} w}{\partial \zeta^{2}}+\left[\zeta+1 / 3+Q_{1}(\zeta)\right] \frac{\partial w}{\partial \zeta} \\
& +R_{1}(x, \zeta)+\beta(1-w)+A\left(\phi^{1 / 2}-1\right) \\
x \frac{\partial \phi}{\partial x}= & \zeta \operatorname{Pr}^{-1} \frac{\partial^{2} \phi}{\partial \zeta^{2}}+\left[\zeta+13 P r+Q_{2}(\zeta)\right] \frac{\partial \phi}{\partial \zeta} \\
& +R_{2}(x, \zeta)+B\left(\phi^{1 / 2}-\phi\right) \\
& +4 C \zeta \phi^{1 / 2}\left(w w_{0}\right)^{-1 / 2}\left(\frac{\partial w}{\partial \zeta}\right)^{2}
\end{aligned}
$$

where

$$
\begin{aligned}
& Q_{1}(\zeta)=-\zeta+1 / 6+\frac{\eta \mathrm{d} \zeta}{4 \mathrm{~d} \eta}-\zeta \frac{\mathrm{d} w_{0}}{\mathrm{~d} \eta} / 4 w_{0} \frac{\mathrm{d} \zeta}{\mathrm{d} \eta}, \\
& Q_{2}(\zeta)=-\zeta+1 / 6 \operatorname{Pr}+\frac{\eta}{4} \frac{\mathrm{d} \zeta}{\mathrm{d} \eta}-\frac{\zeta \frac{\mathrm{d} w_{0}}{\mathrm{~d} \eta}}{4 \operatorname{Pr} w_{0} \frac{\mathrm{d} \zeta}{\mathrm{d} \eta}}+\frac{\zeta}{2 \operatorname{Pr}} \frac{\partial \ln (w / \phi)}{\partial \zeta}
\end{aligned}
$$

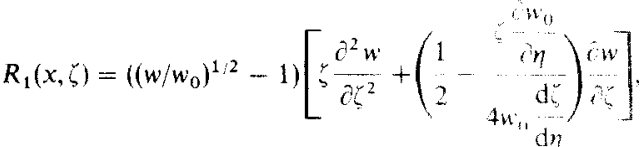

$$
\begin{aligned}
& R_{2}(x, \zeta)=\operatorname{Pr}^{-1}\left(\left(w / w_{0}\right)^{1 / 2}-1\right)\left[\begin{array}{l}
a^{2} \phi \\
\hdashline w^{2}
\end{array}\right.
\end{aligned}
$$

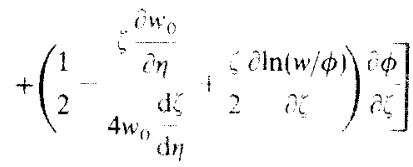

The first three terms on the RHS can be shown to be dominant, as was done in [1]. The choice of representing functions is made by selecting functions which make these terms proportional to the function itsclf. Thus, suitable basis functions are

$$
\gamma_{k}(\zeta)=N_{k} \zeta^{2 / 3} \mathrm{e}^{-\zeta} L_{k-1}^{2 / 3}(\zeta), \quad g_{k}(\zeta)=\hat{\gamma}_{k}(\operatorname{Pr} \zeta)
$$

with

$$
N_{k}=[\Gamma(k) / \Gamma(k+2 / 3)]^{1: 2} .
$$

Here $L$ denotes the generalized Laguer re polynomial, and the $N_{k}$ make the $\gamma_{n}$ and $g_{n}$ orthonormal.

To solve equations (6) and (7). $w$ and $\phi$ are taken to have the forms

$$
w(x, \zeta)=F(x, \zeta)+\sum_{1}^{*} C_{k}(x) \gamma_{k}(\zeta)
$$

$$
\phi(x, \zeta)=I I(x, \zeta)+\sum_{1}^{x} D_{k}(x) g_{k}(\zeta) .
$$

where the functions $F$ and $H$ are chosen so as to improve the rate of convergence of the series solution. Substitution of (12) into (10) and (11), and subsequent use of the ortho-normality relations gives the set of ordinary differential equations

$$
\begin{gathered}
x \frac{\mathrm{d} C_{k}}{\mathrm{~d} x}+k C_{k}=\sum_{m=1}^{\infty} B_{k m} C_{m}+E_{k}, \\
x \frac{\mathrm{d} D_{k}}{\mathrm{~d} x}+k D_{k}=\sum_{m=1}^{x} H_{k m} D_{m}+K_{k},
\end{gathered}
$$

where

$$
\begin{aligned}
& B_{k m}=-\beta \delta_{k m}+\int_{0}^{\infty} \mathrm{e}^{\zeta \zeta-2 \cdot 3} Q_{1}(\zeta) \eta_{k}(\zeta) \frac{\mathrm{d}_{\zeta m}(\zeta)}{\mathrm{d} \zeta} \mathrm{d} \zeta .
\end{aligned}
$$

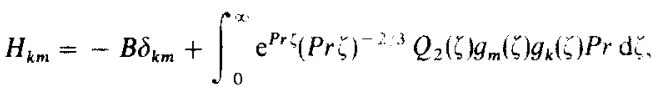

$$
\begin{aligned}
& E_{\mathrm{k}}=\int_{0}^{\mathrm{x}} \mathrm{e}^{\zeta} \zeta^{-2 / 3} \gamma_{k}(\zeta)\left[\zeta \frac{\partial^{2} F}{\partial \zeta^{2}}\right. \\
& +\left(\zeta+1 / 3+Q_{1}(\zeta)\right) \frac{\partial F}{\partial \zeta}+\beta(1-F) \\
& \left.-x \frac{\partial F}{\partial x}+R_{1}+A\left[\left(H+\sum_{1}^{x} D_{m} g_{m}\right)^{1 / 2}-1\right)\right] \mathrm{d} \zeta
\end{aligned}
$$

and

$$
\begin{aligned}
& K_{k}=\int_{0}^{x} \mathrm{e}^{\operatorname{Pr} \zeta}(\operatorname{Pr} \zeta)^{-2 / 3} \operatorname{Prg}_{k}(\zeta)\left\{(\zeta / \operatorname{Pr}) \frac{\partial^{2} H}{\partial \zeta^{2}}\right. \\
& +\left[\zeta+1 / 3 P r+Q_{2}(\zeta)\right] \frac{\partial H}{\partial \zeta}-x \frac{\partial H}{\partial x} \\
& +R_{2}+B\left(\left(H+\sum_{1}^{\infty} D_{m} g_{m}\right)^{1 / 2}-H\right)
\end{aligned}
$$




$$
\begin{aligned}
& +4 C \zeta\left(H+\sum_{1}^{\infty} D_{m} g_{m}\right)^{1 / 2}\left(\frac{\partial F}{\partial \zeta}+\sum_{1}^{\infty} C_{m} \frac{\partial \gamma_{m}}{\partial \zeta}\right)^{2} / \\
& \left.\left[w_{0}\left(F+\sum_{1}^{\infty} C_{m} \gamma_{m}\right)\right]^{1 / 2}\right\} \mathrm{d} \zeta .
\end{aligned}
$$

The integrals appearing in these expressions can be carried out by Gauss-Laguerre quadrature formulae [6]. This was the viewpoint adopted by $\mathrm{Hsu}$ for the constant temperature case. From a knowledge of the asymptotic behavior of the Falkner-Skan solutions, he introduced functions which have the correct behavior for large and small values of $\zeta$, and let $F$ be a linear combination of the initial profile and these functions. In the variable temperature case, the choice of these functions is not as clear, since for Prandtl numbers different from unity, the momentum and thermal boundary layers will have different thickness scales. A reasonable choice, which should at least be satisfactory for values of the Prandtl number near unity, is to choose $F$ and $H$ in the form

$$
F=w_{0}(\zeta)+X_{1}(x) f_{1}(\zeta), \quad H=\phi_{0}(\zeta)+X_{2}(x) f_{2}(\zeta),
$$

where

$$
f_{1}(\zeta)=\frac{1}{6}\left(\frac{9}{4} \zeta\right) \mathrm{e}^{-\zeta}, \quad f_{2}(\zeta)=\frac{1}{6}\left(\frac{9}{4} \zeta\right) \mathrm{e}^{-\operatorname{Pr} \zeta}
$$

The $X$ 's are chosen by expanding $w$ and $\phi$ in the form

$$
w=\sum_{2}^{\infty} \beta_{n}(x) \eta^{n}, \quad \phi=\sum_{2}^{\infty} \alpha_{n}(x) \eta^{n} .
$$

Substitution of these into (6) and (7) leads to recursion relations relating the $\alpha_{n}$ and $\beta_{n}$ to $\alpha_{2}$ and $\beta_{2}$. After some manipulation,

$$
\begin{aligned}
& X_{1}=1.6\left(\beta_{2} / b_{2}-6\right)\left(A_{0}-\beta_{0}\right)+8(A-\beta)\left(b_{2} / \beta_{2}\right)^{1 / 2}, \\
X_{2}= & 0.8\left(A_{0}-\beta_{0}\right)\left(2 \alpha_{0}+3 a_{2}\right) / b_{2} \\
- & 4(A-\beta)\left(b_{2} / \beta_{2}^{1 / 2} \alpha_{2}\right)^{1 / 2} / \beta_{2}+8 \operatorname{Pr}\left(C_{0} b_{2} a_{2}^{1 / 2}-C \beta_{2} \alpha_{2}^{1 / 2}\right) .
\end{aligned}
$$

These expressions depend on knowledge of $\alpha_{2}$ and $\beta_{2}$. This would suggest that the most recent values be used during integration with respect to $\zeta$. Hsu was able to obtain satisfactory results by approximating $\beta_{2}$ as a linear function of $\beta$. The present problem in the general case is more complex because $\alpha_{2}$ and $\beta_{2}$ will depend presumably on $\Phi$ and $U$, as well as their logarithmic derivatives. Even if one assumed a linear dependence of $\alpha_{2}$ and $\beta_{2}$ on the two logarithmic derivatives, two preliminary integrations would be needed to establish the relationship. Only for problems where the variation of the external temperature is sufficiently small will the single pass technique of Hsu work well. Thus it may be just as easy to use results for $\alpha_{2}$ and $\beta_{2}$ at a given $x$ which are extrapolated from the immediately preceding points.

Equations (13) and (14) reduce the problem to a system of first order differential equations of the form

$$
x \frac{\mathrm{d} y}{\mathrm{~d} x}+\lambda y=R(x, y)
$$

Since the system is diagonally dominant, it is stiff and can be solved numerically using a predictor-corrector scheme as given in [5].

A standard forced-convection boundary layer problem is the laminar flow past a circular cylinder. For zero Grashof and Eckert numbers, and a Prandtl number of 0.7, Eckert [7] found that the experiments of Schmidt and Wenner [8] at a Reynolds number of $1.7 \times 10^{5}$ could be approximated by the external velocity

$$
U=2 \theta-0.451 \theta^{3}-0.00578 \theta^{5}
$$

where $\theta=x_{1} / r$ and $r$ is the radius of the cylinder. Choosing the radius as the non-dimensional length $L$, one finds from (5) that

$$
x=\theta^{2}-0.1127 \theta^{4}-0.000964 \theta^{6},
$$

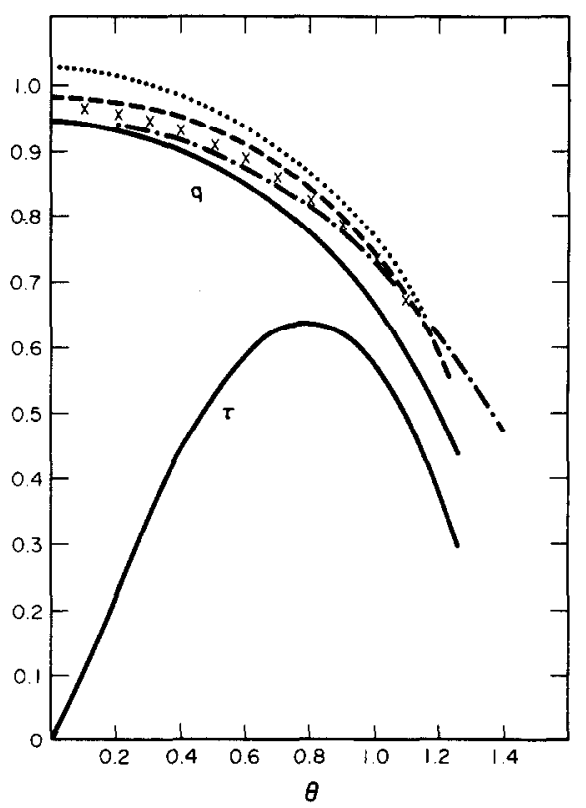

FIG. 1. Wall shear and heat transfer:- - , Frössling [11]; $\cdots$, Hsu case $1[9] ; \times \times \times \times$, Hsu case $2[9] ;---$, Schmidt and Wenner (experimental) [8].

The dimensionless wall shear stress $\tau$, defined by

$$
\tau=\tau_{w} R e^{1 / 2} / \rho U_{\infty}^{2}=U^{2} \beta^{2} / 2 x^{1 / 2}
$$

where $\tau_{w}$ is the true (dimensional) shear stress, and the dimensionless heat flux $q$, defined by

$$
q=\left(\frac{\partial T_{1}}{\partial y_{1}}\right)_{0} L / R e^{1 / 2}\left(T_{\infty}-T_{w}\right)=0.5 U\left(\alpha_{2} \beta_{2} / x\right)^{1 / 2}
$$

were selected as the suitable variables for comparison with previous work. The results of the computations made with $X_{1}$ and $X_{2}$ as linear functions of $\beta$ and with ten terms in each of the series given in equations (12), are shown in Fig. 1. The wall shear agrees well with calculations obtained using a spline fit technique [9], in that the curves appear essentially identical. The present Galerkin method predicts a heat-transfer coefficient which agrees well with those given in [10], [11] for the experimental results up to $x=0.4$, when deviations start to occur.

It appears that the present approach is a useful one for thermal boundary layers, at least for Prandtl numbers near unity. There is more complexity in the initial formulation than for methods which rely heavily on a more direct numerical approach, but the results seem to imply that this complexity may have advantages in the precision of the results and the speed of computation. In any case, the approach seems to be applicable for a broader range of parameters than many of the previously described methods.

Acknowledgements-This work was partially supported by the National Science Foundation under grant number ENG 74-01178.

\section{REFERENCES}

1. C. C. Hsu, A Galerkin method for a class of steady, twodimensional, incompressible, laminar boundary layer flows, J. Fluid Mech. 69, 783-802 (1975).

2. P. A. Libby and H. Fox, Some perturbation solutions in laminar boundary layer theory-Part I. The momentum equation, J. Fluid Mech. 17, 443-499 (1963). 
3. P. A. Libby and H. Fox, Some perturbation solutions in laminar boundary layer theory-Part II. The energy equation, J. Fluid Mech. 19, 433-454 (1964).

4. P. A. Libby, The laminar boundary layer with arbitrarily distributed mass transfer, Int. J. Heat Mass Transfer 9 , 1109-1123 (1966).

5. K. G. Guderley and C. C. Hsu, Numerical integration of the equations for the steady incompressible boundary layer, Aerospace Res. Lab. WPAFB, Ohio, ARL72-0122, AD752-203 (1972).

6. A. H. Stroud and D. Secrest, Gaussian Quadrature Formulas. Prentice Hall, New York (1966)

7. E. R.G. Eckert, Die Berechnung des Wärmeübergangs in der laminar grenz Schicht, ForschH ft. Ver. Dt. Ing. 416, $1-23(1942)$
8. E. Schmidt and K. Wenner, Wärmeabgabe über den Umfang eines angeblasenen geheizten Zylinders, Forsch. Geh. IngWes. 12, 65-73 (1941)

9. C. C. Hsu, The use of splines for the solution of the boundary layer equations, Aerospace Res. Lab. WPAFB, Ohio, Final report on contract F33615-74-C-1116(1975).

10. D. B. Spalding and W. M. Pun, A review of methods for predicting heat-transfer coefficients for laminar uniformproperty boundary layer flows, Int. J. Heat Mass Transfer 5, 239-249 (1962).

11. N. Frössling, Verdunstung, Wärmeübertragung und Geschwindigkeitsverteibing bei zwei dimensionaler und rotationssymmetrischer laminaren Grenzschichtströmung, Lunds. Univ. Arsskr. N. F. Avd. 2, 35 (1940) (English trans. NACA TM 1432).

\title{
A HEAT TRANSFER PREDICTION METHOD FOR TURBULENT BOUNDARY LAYERS DEVELOPING OVER ROUGH SURFACES WITH TRANSPIRATION
}

\author{
P. M. Ligrani, * W. M. Kays and R. J. Moffat
}

Department of Mechanical Engineering, Stanford University, Stanford, CA 94305, U.S.A

(Received 17 March 1980 and in revised form 30 September 1980)

\section{NOMENCLATURE}

$\begin{array}{ll}A, & \text { smooth-wall sublayer thickness; } \\ A^{+}, & A U_{\tau} / v ; \\ A_{R}, & \text { rough-wall sublayer thickness; } \\ A_{R}^{+}, & A_{R} U_{\tau} / v ; \\ C_{f} / 2, & \text { local skin friction coefficient; } \\ C_{p^{1}} & \text { specific heat of fluid; } \\ D^{\prime} & \text { pipe diameter; } \\ F, & \text { blowing fraction; } \\ k_{s}, & \text { equivalent sandgrain roughness; } \\ K_{R}, & \text { fully rough acceleration parameter, } \\ & \left(r / U_{\infty}\right) /\left(\mathrm{d} U_{\infty} / \text { d } x\right) ; \\ l, & \text { mixing length; } \\ l^{+}, & l U_{\tau} / v ; \\ P_{r}, & \text { molecular Prandtl number; } \\ \dot{q}_{w}^{\prime \prime}, & \text { wall heat flux; } \\ r, & \text { radius of spheres comprising test surface; } \\ R, & \text { pipe radius; } \\ R e_{k}, & \text { roughness Reynolds number, } k_{s} U_{\tau} / v ; \\ R e_{D}, & \text { pipe diameter Reynolds number; } \\ R e_{\Delta_{2}}, & \text { enthalpy thickness Reynolds number; } \\ S t, & \text { Stanton number; } \\ \delta t_{0}, & \text { fully rough wall temperature step; } \\ \left(\delta t_{0}\right)^{+}, & \delta t_{0} / T_{\tau} ; \\ T, & \text { mean temperature; } \\ T_{w}, & \text { wall temperature; } \\ T_{\tau}, & \dot{q}_{0}^{\prime \prime} / \rho C_{p} U_{\tau} ;\end{array}$

* Present adddress: von Karman Institute for Fluid Dynamics, Chaussée de Waterloo 72, B-1640 Rhode-St-Genèse, Belgium.

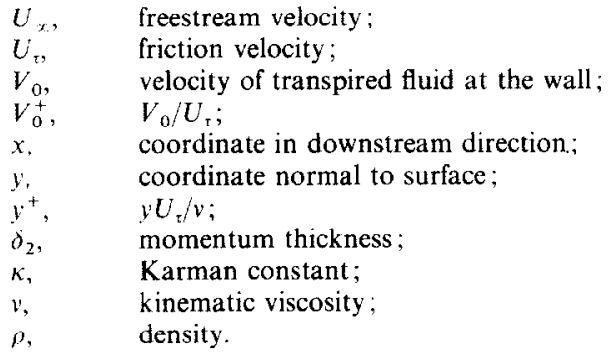

\section{INTRODUCTIO}

THE PURPOSE of the present communication is to present a closure method for the boundary layer equations which can be used to predict Stanton numbers, skin friction coefficients, and mean profiles in boundary layers developing over rough surfaces. The method is the only published one known to the authors for which the combined effects of heat and momentum transfer with both favourable pressure gradient and transpiration may be predicted. Closure is accomplished by specification of mixing-length and turbulent Prandtl number distributions, along with a wall temperature step.

Techniques presently available to predict effects of roughness on turbulent flows are numerous. One of the earliest of these incorporating a mixing-length closure was suggested by van Driest [1]. More recently developed methods range from the integral techniques of Dvorak $[2,3]$ to differential boundary layer methods such as that suggested by Antonia and Wood [4]. Another recent technique is presented by Cebeci and Chang [5], who discuss a differential method with near wall mixing-length equations based on contributions by 